adopted which will enable the owner to obtain full packing value for his cattle, not only would the plan of eradication be more self-supporting (hence it would be more likely to meet with legislative support), but the owner of the diseased cattle would see that it lies in his financial interest to eliminate the tuberculous animals from his herd. Why not permit him to sell his tuberculous cows to some abattoir under inspection? If a cow is shown on postmortem inspection to have only a localized tuberculosis it is worth practically 100 per cent. If the tuberculosis is such that the meat may be canned it is worth about 59 per cent. for its meat, plus an additional sum (approximately $\$ 9.75$ ) for its hide. If so tuberculous that it is rendered into tallow it is worth about 15 per cent., plus the value of the hide.

Should the state wish to add a bonus because of the condemnation, such bonus could be justly placed at a figure below that now given by certain states. Or if a state desires to reimburse to the extent of 30 per cent. for generalized cases and 60 per cent. for localized cases, why not permit the owner to save an additional percentage by which he could reduce his loss still further? It would not be a difficult matter (in case of state reimbursement) to arrange the scale in such a way that the owner would save from 80 to 90 per cent. of the value of the animal. To have a herd free of tuberculosis would be worth some loss on his tuberculous animals.

Finally, the point may be mentioned that if tuberculosis can be eradicated from dairy herds with no, or at most with little, loss to the owner, the increase in the price of milk will, to some extent at least, be inhibited, and the children of poor families will thus be in less danger of having this very important article of their diet decreased.

\section{PERCUSSION OF THE CHEST IN INFANTS AND CHILDREN:*}

\section{SAMUEL MCC. HAMILL, M.D.} PHILADELPHiA.

It seems to be a tendency of the present day to neglect percussion of the chest in early life. This is rather natural, since we find that more valuable, or at least more easily obtainable, results can be secured by auscultation. Furthermore, percussion of the chest in early life is a more difficult procedure and, unless properly done, less satisfactory in its results than is percussion of the adult chest. But, appreciating the valuable assistance one can obtain in the determination of obscure conditions in the thorax by means of properly carried out percussion, I am stimulated to offer this communication as a sort of protest against the neglect of so valuable a procedure.

Successful results from percussion depend on a full knowledge of the subject and its limitations. One must realize the importance of the position of the patient and the necessity of a proper technic and be capable of rightly interpreting the sounds produced by percussion.

\section{PERCESSION IN SOUNDS}

It is not my intention to go into a discussion of the physical qualities of these sounds, nor of the terms used to describe them. Some perception differs so much in different individuals that each will interpret differently the quality of the sounds he elicits on percussion. This, of course, makes no difference in the interpretation of

* Read in the Section on Diseases of Chillren of the American Medical Association, at the lifty-eighth Annual Session, beld at Allantic City, June, 1907. the conditions in the chest, which the various sounds represent, as long as the individual investigator is capable of correctly determining the indications pointed out by the sounds as they are imparted to his ear.

The fewer terms used to describe them the less confusing. For this reason it is best to limit oneself to the following: Tympanitic, hyperresonant, resonant, relatively dull, dull, absolutely dull and flat. Of course, as Sahli expresses it, there is a consecutive series of sound qualities which range in countless gradations between the two limits-resonant and dull-differences depending on the amplitude of the sound vibrations. If desired, these can be indicated by modifications of the above terms.

The sounds produced on percussion depend, for their quality, on the nature of the organ percussed. The resonant sound developed over the normal lung is dependent on two factors: The air contained in the lungthe principal factor-and the vibration of the chest wall.

It was claimed by Williams, Bristowe and others and, to some extent, endorsed by Fagge, that the thorac:c percussion sound was mainly due to the vibrations of the chest wall. In support of this view they pointed out that, without any apparent abnormality in the parietes, deformities of the chest gave rise to absolute du!ness. In lateral curvature of the spine, for instance, the markedly convex projection formed by the ribs on one side of the back, generally, if not alwars. yields a dull sound. This is due to the fact that the peculiar conformation of the chest wall diminishes its vibrating area. The same result is obtained, to a less marked degree, in the normal chest, if percussion is made over the most convex portion of the chest wall just behind the posterior axillary line. It is generally conceded, however, that the percussion sound is chiefly due to the vibrations of the columns of air contained in the organs within the chest wall, but that the wall itself is a contributing factor. In children the chest wall, on account of its greater flexibility, contributes largely, and it is this feature that accounts, in the main, for the distinctly more resonant sound obtained on percussion. of the chest of the child.

\section{CHEST AREAS.}

To percuss the chest of a child satisfactorily, it must be bared. In order to locate the positions of the normal organs, as well as the position of pathologic lesions in these organs, in their relationship to the chest wall, the divisions of the chest wall used in topographic anatomy (supraclavicular and infraclavicular, the mammary, the axillary, the supraspinous, the scalpular, interscapular and infrascapular) and the vertical and horizontal lines are used; (anteriorly the midsternal, the suprasternal and midclavicular; laterally the anterior, middle and posterior axillary lines, and posteriorly the posterior medium and right and left scapular lines and the horizontal mammary).

\section{POSITION}

The only satisfactory position for the percussion of the chest is the one which produces complete relaxation of the muscles and symmetry of its two sides. This is much more difficult to attain and much more important in children than in adults.

In infants and young children the front and anterolateral areas can be examined best with the patient lying flat on his back, with the head in direct contact with the bed, and maintained in the median position 
by the hand of the attendant. The arms should rest at the sides of the body, the thighs and legs should be slightly flexed and the vertebral column should be straight. In this position the child is less resistant and more easily controlled, the body is less apt to sway, muscular relaxation is more complete, the percussion sound is more resonant, and one becomes accustomed to examine in a position which is frequently necessitated by the serious illness of the patient.

It is very easy to convince one's self of the importance of this position by a comparison of the results obtained by changing it, from time to time, during the examination. If, for instance, the head is forcibly turned to one or the other side, as is commonly done, or an arm is abducted until at right angles to the side of the body, one will immediately get a higher pitched sound over the groups of muscles which are thereby brought into a state of tension.

These differences will be greater in some children than in others, being less in those whose general musculature is capable of the greater relaxation, which usually means in those whose attitude of resistance to the examination is least.

For the lateral surfaces (the axillary regions) the position should be the same as for the front of the chest, save that the arms should rest on the bed at right angles to the vertical plane of the body.

The sitting posture is less satisfactory for percussion of both the front and lateral surfaces, because it renders the various chest areas less accessible, the body less fixed, and creates a tendency to push the abdominal organs upward. This is especially the case in children whose stomach and intestines are abnormally distended, or whose livers and spleens are enlarged.

A satisfactory position for the examination of the posterior and postero-lateral surfaces is much harder to secure. Those old enough and not too ill to sit up should be seated on a narrow table or chair, with the legs hanging over its side. The arms should be placed close to the sides of the body, the forearms across the lower abdomen, with the hands resting on the inner surfaces of the thighs. The body should be maintained in a natural stooping posture, without any lateral curving of the spine, and with the head again supported in the median position by the hand of the attendant. Marked flexion or abnormal extension of the body-positions which throw the back muscles into a state of tensionare objectionable. The drooping of one or the other shoulder, which results in tension of the museles and consequent dulling of the resonance on one side or the other, can usually be prevented by the attendant supporting the child, with one hand beneath the occiput and the other over the frontal region.

The turning of the head to one or the other side, or an asymmetrical position of the arms, has the same effect on the percussion sound over the back of the chest as over the front.

To examine the back of an infant it should be held against the chest of the attendant, with its head over her shoulder, its buttocks being supported by her right arm and hand when the head rests on her right shoulder and by her left arm and hand when the head rests on her left shoulder. The vertebral column should be.straight and the two sides made as nearly symmetrical as possible by having the attendant steady the infant by holding its arm with her free hand.

The percussion of the apices is more unsatisfactory than in the adult. They are less well developed, the supraclavicular regions are small and hard to get at, and the isthmus of Krönig is very narrow. To obtain any result in the very young the patient must sit up or be held up and the examiner, standing either in front or behind, may place his thumb or finger in the fossa parallel to the clavicle and pointing toward the sternum.

In older children, and in infants too sick to sit up, or to be removed from the bed, the percussion of the back must be done with the infant lying first on one side and then on the other. In this case the results obtained from percussion of one free side must be compared with the results obtained from percussion of the other free side. To compare the free side with the side in contact with the bed will yield very misleading results, since the compression of the latter largely destroys its vibrating properties, and yields, therefore, a dull or sometimes flat sound.

I have frequently seen students locate the consolidation of a pneumonic process on the wrong side by percussing the chest of an infant with pneumonia while it lay on the unaffected side.

As a further illustration of the misleading effect of an incorrect position, I saw a mistaken diagnosis made, a few years ago, in a very important case by a very able clinician. He was called in consultation, to confirm the opinion which had been expressed by two pediatriststhat the infant did not have pneumonia. When he examined the child the youngster was fretful and resisted the examination in such a way as to throw the muscles on the left side of the chest, which was next to him, into contraction. Percussion over this area yielded a dull, almost flat, sound, while the opposite side was resonant. Auscultation over this dulled area revealed a relatively feeble respiratory murmur. The signs seemed so definite that a diagnosis of pneumonia was promptly and unhesitatingly made. As soon as the consultant left the house the child was placed on the opposite side of the bed, and as he resisted the examination in the same manner, in relation to the other side, a simliar dulled sound was readily elicited over the right chest, while the side which had formerly been dull was entirely resonant. I mention this incident to impress on your minds the fact that even a careful, shrewd diag. nostician may fall into very serious error unless he takes into consideration the peculiar influences of position on the sounds produced by percussion.

\section{TECHNIC.}

As to the technic of percussion, the best results can be obtained in young children by the finger-finger method. Instruments are apt to alarm, they are not as adaptable to the different portions and small size of the chest wall and they eliminate the palpatory element of the finger-finger method, which is of especial value in early life. In this method the distal phalanx of the middle finger forms the pleximeter. Its palmar surface is placed against the chest wall with moderately firm and even pressure. This limited area of contact of the finger with the chest wall avoids too wide diffusion of the sound when the blow is struck, and the moderately firm, even pressure avoids the dulling effect of a loosely applied finger and the discomfort of too great pressure.

The amount of pressure must be the same each time the finger is applied to the chest, in order to insure a satisfactory comparison of the sounds produced in striking corresponding portions of its two sides.

The older teaching, and the method which still scems to be largely used, of placing the full length of the fin- 
ger against the chest wall and percussing over the middle phalanx, certainly should have no place in the percussion of the child's chest. Too much of the surface of the chest is covered, in consequence of which the resulting sound is not produced by the conditions immediately underlying the point over which the percussion is made, but represents the condition of the parietes underlying the entire length of the finger. A limited area of consolidation, such as one often finds in bronchopneumonia, for instance, might be very easily lost in the resonant sound transmitted through the fingers from the adjacent areas of healthy lung. Indeed, to reduce the point of contact of the pleximeter finger to the minimum the recommendation of Plesch-that the best results in percussion can be obtained by flexing the finger at the first interphalangeal joint, applying the tip of the finger to the area to be percussed, and delivering the blows on the finger thus flexed-is well worthy of consideration by those whose finger joints are flexible enough to admit of this manipulation.

It is not essential, in examining infants and young children, to place the finger parallel to the ribs, first, because it is impossible to get the finger into the interspaces, and second, because the flexibility of the ribs is so great that they have very little deadening effect on the sound. As a working basis, however, it may be better to follow out this established method.

The percussion movement should be from the wrist, not from the elbow; the tip of but one finger should be used as a plexor and it should strike the pleximeter finger perpendicularly. The strokes should be equal in force for each comparison, not made too rapidly nor repeated too frequently, and the percussing finger should remain in only momentary contact with the percussed finger. Percussion with the wrist joint stiff and the finger at an obtuse angle dulls the sound, makes the patient uncomfortable and the stroke inaccurate. Rapid and frequently repeated strokes confuse the ear, and prolonged contact of the striking and struck fingers reduces the vibration of the chest wall and dulls the sound.

The most important part of the procedure relates to the force of the stroke. It should always be light-the minimized stroke of Gairdner-because the hard stroke is alarming and painful to the patient, and because, as Gairdner has pointed out, "percussion does not operate directly downward, nor in the direction of the impact only, but in proportion to the strength of the stroke laterally, diagonally and in every possible direction." Consequently, any area of circumscribed dulness may easily be lost in the transmitted resonance from surroundng healthy lung tissue if the stroke is too strong.

The advantages of this light stroke can not be too strongly emphasized, and when I speak of light percussion, I mean very light percussion, percussion that will produce a sound that is audible only a very short distance from the patient. I have no difficulty in persuading my students of its value and I am convinced that anyone who will give it a careful trial will appreciate its importance.

I do not mean to deny that there are instances in which valuable results may be obtained by using stronger percussion; indeed, it is very important, under certain conditions, to vary the force of the percussion stroke, as it is also important at times to vary the degree of pressure of the finger applied to the chest wall, but the point which I want to make is that, for ordinary percussion, the light stroke is far superior to the strong one, and that it is the one which we should recommend to our students.

\section{CONDITIONS WHICH INTERFERE.}

In addition to a defective position of the patient, and a poor technic, there are certain other influences which are apt to yield misleading results. For instance, the act of respiration. The sound produced at the end of inspiration, when the chest is tense, notwithstanding the increased amount of air in the lung, is higher in pitch than the sound elicited at the end of expiration, when the muscles are relaxed and the chest wall more flexible.

The fixed position of the chest wall during the act of crying dulls the resonance, especially in the lower posterior portion of the thorax, but there is probably nothing which so effectually interferes with one's results as the presence of an abdomen distended with gas. This effect may be unilateral or bilateral, depending on which of the abdominal viscera are distended. The presence of gas in the stomach, for instance, may produce a tympanitic note throughout the entire left chest, while the distension of the colon or both colon and stomach may produce a tympanitic sound throughout the entire chest. Most commonly, of course, the lower posterior portions are affected, and more often, on account of the protecting power of the liver, the left than the right. This tympany is very misleading, and we have all doubtless heard the dull sound of a consolidated lung entirely obliterated by the transmitted tympanitic sound from a distended stomach or gut.

I am at a loss to advise you as to a satisfactory method of percussion under these conditions, but I am inclined to believe that the lateral limitation method of Schott is sometimes helpful. This consists in placing three fingers of the left hand on the chest wall and percussing over the middle phalanx of the third finger. It limits the lateral vibration of the chest wall and, as the tympanitic sound is probably largely transmitted through the medium of the chest wall, its transmission is thereby partly controlled.

There is another point which is well worthy of consideration in percussion of the lungs, and that is the relationship of the patient to the walls of the room in which he is examined. If he is placed close to the wall, with one side of the body next to it, the percussion sound on that side will be more resonant than on the opposite side. If he is placed in the corner of a room with the two sides of the body equidistant from the walls, the resonance over both sides, as has been pointed out by Dr. Brown of Saranac, will be enormously increased. In the light of these facts, therefore, it is well not to have the patient too close to the walls of the room while the chest is being percussed.

There are so many conditions which influence the results of percussion, especially in the child, that one has no right to accept any area of dulled resonance as indicative of changes within the chest wall until all possible external influences have been eliminated.

THE SOUNDS OF THE NORMAL CHEST.

The sound elicited on percussion over the lung of the normal infant, or young child, that is, one in whom there is not only a normal condition of the chest wall, but also of the organs within the thorax, and a normal condition and position of the abdominal organs, is much more resonant than the sound produced by percussion over the lung of a normal adult. The percussion of the front of the chest yields the following result: On the right-from the clavicle to the fourth rib-one elicits 
the full normal pulinonary resonance of the child. Below this point, owing to the decreased volume of lung and the presence behind it of the liver, the sound becomes gradually dull and finally passes into the dull sound of the liver at the sixth rib. The anterior borders of the lungs do not extend under the sternum, in consequence of which there is no pulmonary resonance in this area. This latter and the increased volume of the percussion sound represent the only differences from the adult chest.

On the left side, however, the differences are more marked. The principal one is the presence of a relatively dulled area behind and beneath the inner third of the clavicle, which sometimes extends outward to the midclavicular line, and always downward until it fades into the cardiac dulness. This is apparently dependent on the posterior position of the lung and the consequent proximity of the great vessels. The existence of this relatively dulled area is not only not referred to in the text-books, but, on the contrary, we are usually tanght that the percussion sound over the right upper chest is somewhat duller than over the left. Unless, therefore, one bears in mind the existence of this area, one is very apt, as I have observed, to interpret it as a pathologic condition.

Without the midclavicular line, and above the transverse mammary line, the resonance corresponds closely to that of the same area on the opposite side. Below this point the sound becomes tympanitic, due to the proximity of the stomach. Over the balance of the front of the left chest the sound varies from relatively dulled to dull, and will be referred to later in considering the percussion of the heart.

Latcrally, on both sides, there is good resonance above the sixth rib. Below this point, on the right, the underlying liver produces a relatively dulled to dull sound, while on the left side the stomach yields a tympanitic sound.

Posteriorly the percussion sound is everywhere more resonant than over the back of the adult chest, because the muscles are less developed and the ribs and scapulæ more flexible. The most resonant areas are the infrascapular and interscapular regions; the next most resonant, the lower scapular; and the least resonant, the upper scapular and supraspinous regions. The dulling of the sound in the lower right infrascapular region, due to the proximity of the liver, marks the only difference in the two sides. The convex portion of the thorax on each side, just posterior to the posterior axillary line, has a decided dulling effect on the resonance, and to a much greater degree than in the adult chest.

The sounds over the apices, owing to the relatively poor development of this part of the.lung, are relatively less resonant than in the adult.

PERCUSSION OF THE HEART.

In early life the heart occupies a somewhat different position in the thoracic cavity than in adult life. It is situated higher in the chest and more horizontally. This position is probably dependent on the size of the heart, the undeveloped condition of the lungs, the recumbent position in which the child spends much of its time, the less strenuous life which it leads and the higher position of the diaphragm.

In the infant the apex beats in the fourth space in, or just without, the midclavicular line. As the child develops there occurs a gradual change in the position of the anex. In this process of change it first descends in an almost perpendicular direction to the fifth space. It then gradually moves inward until it reaches the normal adult position, at a more or less indefinite period between the third and twelfth years.

It is readily manifest, when one realizes the position of the heart within the thorax in early life, that the relationship of the heart to the chest wall is necessarily different from that of the adult heart.

The entire heart is higher, in consequence of which the upper border is elevated and lies back of the second rib. The more horizontal position causes a relatively greater transwerse diameter, which brings the right border to or a little beyond the right edge of the narrower sternum and the left, just without the midclavicular line.

As to the best method of determining these outlines by percussion, there is and always has been a great diversity of opinion. Many authorities advise against any attempt to determine the actual size of the heart. but prefer to determine its normality or abnormality by developing what is known as the absolute or superficial heart dulness; that is, the outlining by percussion of that portion of the heart not covered by lung and which is, therefore, in more direct contact with the chest wall. Others, and probably the majority, believe that more accurate information will result from attempting to determine the actual size of the heart by bringing out what is called the deep cardiac dulness. Still others use both procedures. Without going into the arguments advanced for and against these two methods, I would say that, in so far as children are concerned, I am in accord with those who believe that the most uniform and accurate results can be obtained by determining the actual size of the heart.

In infants the outlines can be determined best with the patient in the recumbent posture. In fact, in childre:I at all ages, this position will yield more accurate results than can be obtained when the patient is upright.

The apex beat and the outlines are practically the same in the two positions in the very young. But even admitting that differences should occur, it really does not affect one's conclusions, provided one always percusses the heart with the patient in the recumbent posture.

Finger-finger percussion is preferable to instruments in outlining the heart. The stroke must be extremely light, even lighter than that used in the percussion of the lungs; so light, in fact, that the sound produced can not be heard unless the ear is held close to the chest wall. As Rosenbach says: "The object is to elicit the most delicate shades of sound. The softer the sound, the less distinct will be the independent sound of the chest wall or of the lung, and the more distinct will be the sound of the mass of the heart."

Goldscheider, who also advocates this method, says that this light stroke necessarily reduces the transverse extension of the sound, and that "the axial extension into the depths of the tissue greatly exceeds the transverse extension." To apply this light stroke properly, noise must be eliminated so far as possible, and one must know what sort of sounds will result. If one percusses toward the border of the heart, from resonant lung, the sound which is always slight will change abruptly when the deep borders of the heart are reached, from a light resonance to an almost imperceptible sound, and unless the stroke is light enough to produce this result the method is improperly applied.

As in lung percussion, only the distal phalanx must 
be applied to the chest wall and the amount of pressure must be the same. 'The finger must be placed parallel to the particular border of the heart which is being outlined, and the stroke must always be made at right angles to the pleximeter finger and in the anteroposterior direction. This latter point is of the utmost importance, especially in determining the lower left border of the heart. In the normal chest the curve of the ribs becomes marked as the midclavicular line is approached. There is a natural tendency, therefore, as the left cardiac border is reached, to follow the shape of the chest and percuss against the left border rather than parallel to it. It is very manifest that this procedure will carry the left border of the heart much beyond its actual position, especially in those whose chest walls are thin, and particularly in rachitic children, in whom there is a marked drawing in of the lower anterolateral borders of the chest.

The application of the full length of the finger to the chest wall, with percussion over the middle phalanx, is especially objectionable in outlining the hearts of infants and children. As the extreme lower border of the heart is approached, for instance, the finger thus applied will cover resonant lung above, heart in its middle portion, and liver and stomach in their lower portions. The mixed sound resulting from such percussion is obviously too misleading to be of any practical use in outlining this part of the heart.

If the proper method is pursued, the left border of the heart is more easily determined than either the upper or the right. It is only when there is marked distension of the abdomen that this becomes difficult, and under these conditions, the full value of the light stroke will be fully appreciated.

The area of impaired resonance beneath the inner third of the left clavicle, which varies in different individuals, sometimes renders the determination of the upper border extremely difficult. Usually, there is a shading off to a less dulled sound, in percussing from above downward, before the cardiac dulness is reached, which enables one to determine the upper border of the heart more or less readily.

The bony resonance of the sternum and the higher position of the diaphragm and liver render the outlining of the lower right border difficult. In rachitic children with enlargement of the liver and chondrosternal thickening, its accurate determination is sometimes almost impossible.

Respiration unquestionably influences the result, but unfortunately, it is impossible in young children to control the respiratory movements as one can in the adult. One should make the stroke at the end of expiration, however, in so far as it is possible. The heart is then less covered by lung and in closer contact with the chest wall.

There is only one method by which the actual size of the heart can be determined, and that is the orthodiagraphic method of Moritz, which is of no value as a routine measure, because of the difficulty of its application and of the dangers attendant on the common use of the screen in $x$-ray work. Its discovery is clever, however, and it has its place in medicine as a measure by which we can determine which of the commonly used methods of outlining the heart is the most accurate.

The difficulty of the application of the $x$-ray to the determination of the size of the heart lay in the fact that, owing to the divergence of the Roentgen rays, the shadow cast by a body does not correspond to its true shape and size, but is enlaryed and partly blurred. Moritz, in establishing his orthodiagraphic method, constructed an apparatus with which the outline of an object can be taken with parallel rays running vertically to the transillumination screen. He found it impossible to keep the entire bundle of Roentgen rays parallel, and, to overcome this, he employed, for making the projection, only that ray which strikes the transillumination screen at right angles, by placing the tube in a plane parallel to the transillumination screen, at the same time moving the marked vertical ray so that it is parallel to itself. In this manner he has succeeded in tracing the actual size of the heart.

The method has been applied extensively in Germany, mainly for the purpose of confirming the accuracy of the various methods of cardiac percussion. The results tend to show that the determination of the deep cardiac dulness is more accurate as an indication of the actual size of the heart and that the best results are obtained by the use of a very light percussion stroke. Orthodiagraphy has not been applied to the determination of the size of the infant's or young child's heart, and it would probably be attended with considerable difficulty, since the perfect control of the patient is an important factor.

I have been unable to persuade any of the Roentgenologists with whom I am associated to apply the method on account of their objection to the use of this screen, which has been largely responsible for the majority of the fatalities among the $x$-ray workers.

Note.-Since I wrote the above paper, I find that Hænisch, in a communication before the German Roentgen Society, reports the employment of the orthodiagraphic method radiographically, with the result of accurately estimating the size of the heart. In the discussion of this communication, Schoenlerg made the statement that he had also found the method absolutely accurate.

\section{DISCUSSION.}

Dr. G. H. Cattermole, Boulder, Colo, thought that more attention should be given to the tactile sense during percussion. In children who are crying one can often get considerable information from the sense of touch in the finger used as a pleximeter.

Dr. H. Lowenburg, Philadelphia, stated that, although he has tried to find this relative area of dulness below and to the inner third of the left clavicle, he has been unable to satisfy himself of its existence. He asked Dr. Hamill to explain the reason why this relative area of dulness occurs.

Dr. J. H. MCKEE, Philadelphia, said that he had been able to confirm in nearly every instance what Dr. Hamill said as to the dulness under the left clavicle and along the left border of the sternum. He also endorsed what he said of light percussion.

Dr. A. W. Fairbanks, Boston, endorsed the value of auscultatory percussion. He has found it invaluable in the detection of slight variations in quality and pitch. He uses a Snofton stethoscope, unscrewing the hard rubber bell and using the small metal end. For children, especially, he has found this method exceedingly effective, particularly in detecting small areas of consolidation.

Dr. Alfred Hand, Jr., Philadelphia, realized that he had a tendency to overlook the use of percussion to some extent. The results, he said, are certainly not so valuable as those obtained by auscultation. Thrs is in large part due to the personal equation of the observer, the ability of different ears to recognize musical changes of pitch. He has often observed this in trying to elicit changes in the percussion note in hospital practice, by getting expressions of opinion from the resident physician and the nurse, and sometimes has had three different interpretations of the sound. 